\title{
The critical surface fugacity for self-avoiding walks on a rotated honeycomb lattice
}

\author{
Nicholas R. Beaton \\ LIPN (UMR 7030), Université Paris 13, 93430 Villetaneuse, France
}

\begin{abstract}
In a recent paper with Bousquet-Mélou, de Gier, Duminil-Copin and Guttmann (2012), we proved that a model of self-avoiding walks on the honeycomb lattice, interacting with an impenetrable surface, undergoes an adsorption phase transition when the surface fugacity is $1+\sqrt{2}$. Our proof used a generalisation of an identity obtained by Duminil-Copin and Smirnov (2012), and confirmed a conjecture of Batchelor and Yung (1995). Here we consider a similar model of self-avoiding walk adsorption on the honeycomb lattice, but with the impenetrable surface placed at a right angle to the previous orientation. For this model there also exists a conjecture for the critical surface fugacity, made by Batchelor, Bennett-Wood and Owczarek (1998). We adapt the methods of the earlier paper to this setting in order to prove the critical surface fugacity, but have to deal with several subtle complications which arise.
\end{abstract}

This article is an abbreviated version of a paper of the same title, currently being prepared for submission.

Résumé. Dans un article récent avec Bousquet-Mélou, de Gier, Duminil-Copin et Guttmann (2012), nous avons prouvé qu'un modèle de marches auto-évitantes sur le réseau hexagonal, interagissant avec une surface impénétrable, subit une transition de phase absorbante quand la fugacité de la surface est $1+\sqrt{2}$. Notre preuve utilisait une généralisation d'une identité obtenue par Duminil-Copin et Smirnov (2012), et permettait d'établir une conjecture de Batchelor et Yung (1995). Ici nous considérons un modèle similaire d'absorption de marches aléatoires auto-évitantes sur le réseau hexagonal, mais avec une surface impénétrable placée à angle droit par rapport à l'orientation précédente. Pour ce modèle il existe aussi une conjecture concernant la fugacité critique de la surface, formulée par Batchelor, Bennett-Wood et Owczarek (1998). Nous adaptons les méthodes de l'article précédent à ce cadre afin de prouver la fugacité critique de la surface, mais devons faire face à plusieurs complications subtiles qui apparaissent.

Cet article est la version courte d'une article ayant le même titre et actuellement en préparation.

Keywords: self-avoiding walks, polymer adsorption, honeycomb lattice, discrete holomorphicity

\section{Introduction}

Self-avoiding walks (SAWs) have been considered a model of long-chain polymers in solution for a number of decades - see for example early works by Orr (1947) and Flory (1949). In the simplest model one associates a weight (or fugacity) $x$ with each step (or monomer, in the context of polymers) of a walk, and then (for a given lattice) considers the generating function

$$
C(x)=\sum_{n \geq 0} c_{n} x^{n},
$$

1365-8050 @ 2013 Discrete Mathematics and Theoretical Computer Science (DMTCS), Nancy, France 
where $c_{n}$ is the number of SAWs starting at a fixed origin and comprising $n$ steps.

It is straightforward to show (see e.g. Madras and Slade (1993)) that the limit

$$
\mu:=\lim _{n \rightarrow \infty} c_{n}^{1 / n}
$$

exists and is finite. The lattice-dependent value $\mu$ is known as the growth constant, and is the reciprocal of the radius of convergence of the generating function $C(x)$. The honeycomb lattice is the only regular lattice in two or more dimensions for which the value of the growth constant is known; its value $\mu=\sqrt{2+\sqrt{2}}$ was conjectured in 1982 by Nienhuis (1982) and proved by Duminil-Copin and Smirnov (2012).

The interaction of long-chain polymers with an impenetrable surface can be modelled by restricting SAWs to a half-space, and associating another fugacity $y$ with vertices (or edges) in the boundary of the half-space which are visited by a walk. It is standard practice to place the origin on the boundary. This naturally leads to the definition of a partition function

$$
C_{n}^{+}(y)=\sum_{m \geq 0} c_{n}^{+}(m) y^{m},
$$

where $c_{n}^{+}(m)$ is the number of $n$-step SAWs starting on the boundary of the half-space and occupying $m$ vertices in the boundary.

The limit

$$
\mu(y):=\lim _{n \rightarrow \infty} C_{n}^{+}(y)^{1 / n}
$$

has been shown to exist for the $d$-dimensional hypercubic lattice for $y>0$ (see e.g. Hammersley et al. (1982)). It is a finite, log-convex and non-decreasing function of $y$, and is thus continuous and almost everywhere differentiable. The adaptation of the proof to other regular lattices (in particular, to the honeycomb lattice) is elementary - see Beaton (2012) for details.

It can also be shown that for $0<y \leq 1$,

$$
\mu(y)=\mu(1)=\mu,
$$

and that $\mu(y) \geq \max \{\mu, \sqrt{y}\}$. (The lower bound $\sqrt{y}$ applies to the honeycomb lattice as discussed in this paper, but this bound varies depending on the lattice and orientation of the surface $\left.{ }^{(i)}\right\}$ This implies the existence of a critical fugacity $y_{\mathrm{c}} \geq 1$ satisfying

$$
\mu(y) \begin{cases}=\mu & \text { if } y \leq y_{\mathrm{c}}, \\ >\mu & \text { if } y>y_{\mathrm{c}} .\end{cases}
$$

This critical fugacity signifies an adsorption phase transition, and demarcates the desorbed phase $y<y_{\mathrm{c}}$ and the adsorbed phase $y>y_{\mathrm{c}}$.

Just as the honeycomb lattice is the only regular lattice whose growth constant is known exactly, it is also the only lattice for which an exact value for $y_{\mathrm{c}}$ is known. In fact, because there are two different ways to orient the surface (see Figure 1) for the honeycomb lattice, there are two different values of $y_{\mathrm{c}}$. When the

(i) In general, it is straightforward to show $\mu(y) \geq y^{1 / k}$, where $k$ is the minimum number of steps required to walk from one weighted vertex to another. 


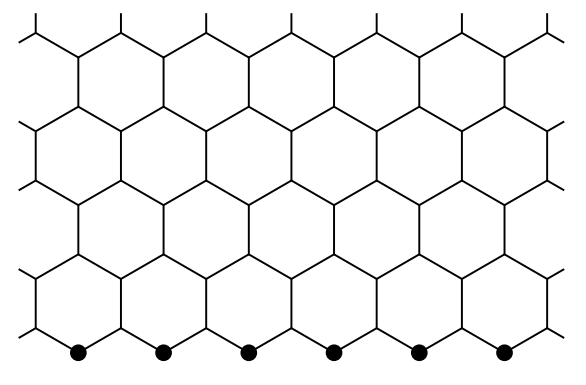

(a)

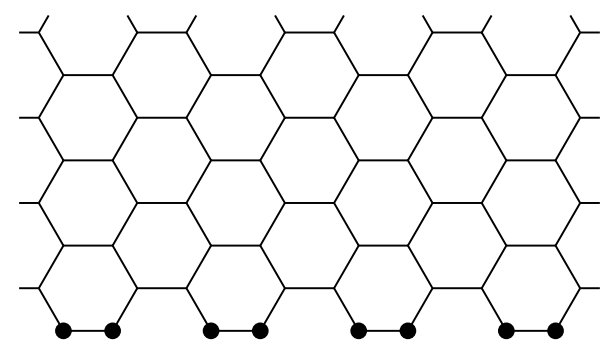

(b)

Fig. 1: The two orientations of an impenetrable surface on the honeycomb lattice, with the surface vertices indicated.

surface is oriented so that there are lattice edges perpendicular to the surface (i.e. Figure 1(a)), the critical fugacity is $y_{\mathrm{c}}=1+\sqrt{2}$. This value was conjectured by Batchelor and Yung (1995), using the integrability of the model and comparison with a more general solvable loop model on the square lattice. The critical boundary weight was obtained by finding reflection matrices which satisfy the boundary Yang-Baxter equation. A proof was discovered by Beaton et al. (2012); it used a generalisation of an identity obtained by Duminil-Copin and Smirnov (2012), as well as an adaptation of some results of Duminil-Copin and Hammond (2012).

It is the other orientation of an impenetrable surface on the honeycomb lattice (i.e. Figure 1 (b)) that is the focus of this article. For this model of polymer adsorption there is also a conjecture regarding the critical surface fugacity, due to Batchelor et al. (1998) and obtained using the same methods as for the first orientation. In this extended abstract we sketch the proof of that result:

Theorem 1 For the self-avoiding walk model on the semi-infinite honeycomb lattice with the boundary oriented as per Figure $1 \mathrm{~b}$ ), the critical surface fugacity is

$$
y=y_{\mathrm{c}}=\sqrt{\frac{2+\sqrt{2}}{1+\sqrt{2}-\sqrt{2+\sqrt{2}}}}=2.455 \ldots
$$

This paper is an overview of Beaton (2012), which in turn largely follows the same structure as Beaton et al. (2012). In the interest of brevity we omit most proofs. We first present an identity relating several different generating functions of SAWs in a finite domain, evaluated at the critical step fugacity $x=x_{\mathrm{c}}=$ $\mu^{-1}$. We then give adaptations of some existing results for the hypercubic lattice to the honeycomb lattice, and show how the critical fugacity relates to an appropriate limiting case of our identity. This relationship enables us to derive a proof of Theorem 1, subject to a certain generating function in a restricted geometry (specifically, the generating function of self-avoiding bridges which span a strip of height $T$ ) disappearing in a limit. We omit the proof of that result here; it is given in the appendix of Beaton (2012). The proof there is very similar to that of the appendix in Beaton et al. (2012), which was in turn based on arguments featured in Duminil-Copin and Hammond (2012).

In Beaton et al. (2012), we also established identities for a generalisation of the self-avoiding walk model, namely the $O(n)$ loop model. The equivalent generalisation for the rotated lattice is discussed in Beaton (2012), and we refer the reader to that article for further details. 


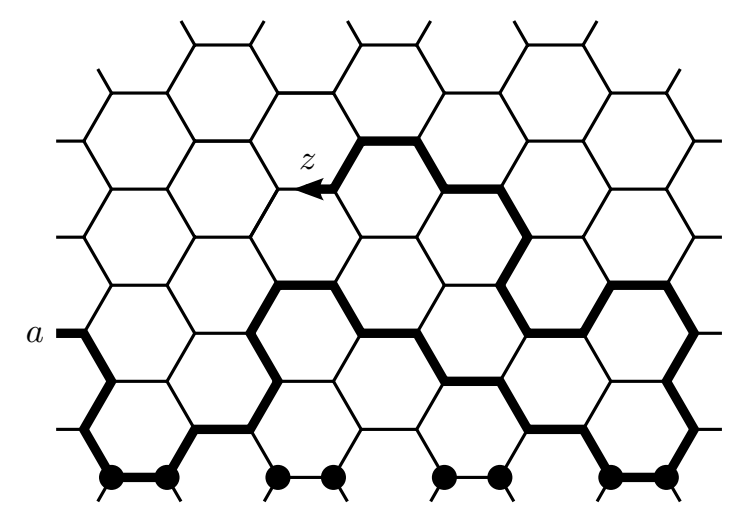

Fig. 2: A SAW on the honeycomb lattice. The contribution of this SAW to $F(z)$ is $\mathrm{e}^{-\mathrm{i} \sigma \pi} x^{33} y^{4}$.

\section{The identities}

\subsection{The local identity for bulk vertices}

We consider the semi-infinite honeycomb lattice, oriented as in Figure 1 1 b), embedded in the complex plane in such a way that the edges have unit length. We follow the examples of Duminil-Copin and Smirnov (2012) and Beaton et al. (2012) and consider self-avoiding walks which start and end at the mid-points of edges on the lattice. Note that this means the length of a walk is the same as the number of vertices it occupies. We define a domain $\Omega$ to be a finite connected collection of mid-edges with the property that for every vertex $v$ adjacent to a mid-edge of $\Omega$, all three mid-edges adjacent to $v$ must be in $\Omega$. We denote by $V(\Omega)$ the set of vertices adjacent to mid-edges of $\Omega$, and by $\partial \Omega$ the set of mid-edges of $\Omega$ adjacent to only one vertex of $V(\Omega)$. Let $\gamma$ be a self-avoiding walk. We denote by $|\gamma|$ the number of vertices occupied by $\gamma$ and by $c(\gamma)$ the number of contacts with the surface (i.e. vertices on the surface occupied by $\gamma$ ).

Now define the following so-called parafermionic observable: for $a \in \partial \Omega$ and $z \in \Omega$, set

$$
F(\Omega, a, p ; x, y, \sigma) \equiv F(p):=\sum_{\gamma: a \rightarrow p} x^{|\gamma|} y^{c(\gamma)} \mathrm{e}^{-\mathrm{i} \sigma W(\gamma)},
$$

where the sum is over all SAWs $\gamma \subset \Omega$ which run from $a$ to $p$, and $W(\gamma)$ is the winding angle of $\gamma$, that is, $\pi / 3$ times the difference between the number of left turns and right turns. See Figure 2 for an example.

The following lemma appears as part of Lemma 3 in Beaton et al. (2012); the case $y=1$ is due to Smirnov (2010).

Lemma 2 Let

$$
\begin{aligned}
\sigma & =-\frac{1}{8}, & x_{\mathrm{c}}^{-1} & =2 \cos \left(\frac{3 \pi}{8}\right)=\sqrt{2-\sqrt{2}}, \\
\sigma & =\frac{5}{8}, & x_{\mathrm{c}}^{-1} & =2 \cos \left(\frac{\pi}{8}\right)=\sqrt{2+\sqrt{2}} .
\end{aligned}
$$


Then for a vertex $v \in V(\Omega)$ not belonging to the weighted surface, the observable $F$ satisfies

$$
(p-v) F(p)+(q-v) F(q)+(r-v) F(r)=0,
$$

where $p, q, r$ are the three mid-edges adjacent to $v$, and the variable $x$ is set to $x_{\mathrm{c}}$.

Equation (1) corresponds to the larger of the two critical values of the step weight $x$ and hence to the dense regime critical point, while 22 corresponds to the line of critical points separating the dense and dilute phases. In what follows we refer to (1) and (2) as the dense and dilute regimes respectively.

\subsection{The local identity for surface vertices}

We now wish to generalise Lemma 2 to include vertices lying on the weighted boundary. To do this, we have to be more particular about the domain being used. We work in the special domain $D_{T, L}$, as illustrated in Figure 3. The height $T$ of the domain is the length of the shortest walk starting at $a$ and ending at the top boundary; the width $2 L+1$ is the number of columns of cells. Walks start at the midedge $a$. We choose this mid-edge in order to preserve the reflective symmetry of the domain, which greatly simplifies an important identity. However, the fact that $a$ is not an external mid-edge does introduce some complications:

- A walk which ends at a particular external mid-edge could have two different winding angles, depending on whether it started from $a$ in the left or right direction. This is undesirable, but easily corrected. Define

$$
W^{*}(\gamma):= \begin{cases}W(\gamma)+\pi / 2 & \text { if } \gamma \text { starts in the left direction, } \\ W(\gamma)-\pi / 2 & \text { if } \gamma \text { starts in the right direction, } \\ 0 & \text { if } \gamma \text { is the empty walk. }\end{cases}
$$

Then, define $F^{*}(p)$ in the same way as $F(p)$, but now using $W^{*}$ instead of $W$.

- This new observable $F^{*}$ will satisfy the same identity $(3)$ as $F$ on all non-boundary vertices of $D_{T, L}$, except for the vertices $a^{-}$and $a^{+}$adjacent to $a$. To deal with this, we define $V^{\prime}\left(D_{T, L}\right):=$ $D_{T, L} \backslash\left\{a^{-}, a^{+}\right\}$, and will end up evaluating (3) only on the vertices of $V^{\prime}\left(D_{T, L}\right)$.

Proposition 3 Let $\sigma$ and $x_{\mathrm{c}}$ be as defined in (2). Define $\mathbf{1}_{\beta^{+}}(v)$ to be 1 if the vertex $v$ is adjacent to a mid-edge in $\beta^{+}$and 0 otherwise, and similarly define $\mathbf{1}_{\beta^{-}}(v)$. Then for every vertex $v$ in $V^{\prime}\left(D_{T, L}\right)$ with adjacent mid-edges $p, q, r$,

$$
\begin{aligned}
& (p-v) F^{*}(p)+(q-v) F^{*}(q)+(r-v) F^{*}(r) \\
& =\mathbf{1}_{\beta^{+}}(v)(1-y) \mathrm{e}^{-\mathrm{i} \sigma(-\pi / 6)}\left(x_{\mathrm{c}} y\right)^{-1}\left((r-v) \bar{\lambda} \sum_{\gamma: a \rightarrow r \rightarrow p} x_{\mathrm{c}}^{|\gamma|} y^{c(\gamma)}+(q-v) \lambda \sum_{\gamma: a \rightarrow q \rightarrow p} x_{\mathrm{c}}^{|\gamma|} y^{c(\gamma)}\right) \\
+ & \mathbf{1}_{\beta^{-}}(v)(1-y) \mathrm{e}^{-\mathrm{i} \sigma(\pi / 6)}\left(x_{\mathrm{c}} y\right)^{-1}\left((r-v) \bar{\lambda} \sum_{\gamma: a \rightarrow r \rightarrow p} x_{\mathrm{c}}^{|\gamma|} y^{c(\gamma)}+(q-v) \lambda \sum_{\gamma: a \rightarrow q \rightarrow p} x_{\mathrm{c}}^{|\gamma|} y^{c(\gamma)}\right),
\end{aligned}
$$

where for vertices adjacent to mid-edges in $\beta^{+}$or $\beta^{-}$, the surrounding mid-edges $p, q, r$ are in clockwise order from the external mid-edge, and the sums are over walks which visit the indicated mid-edges in the prescribed order. 


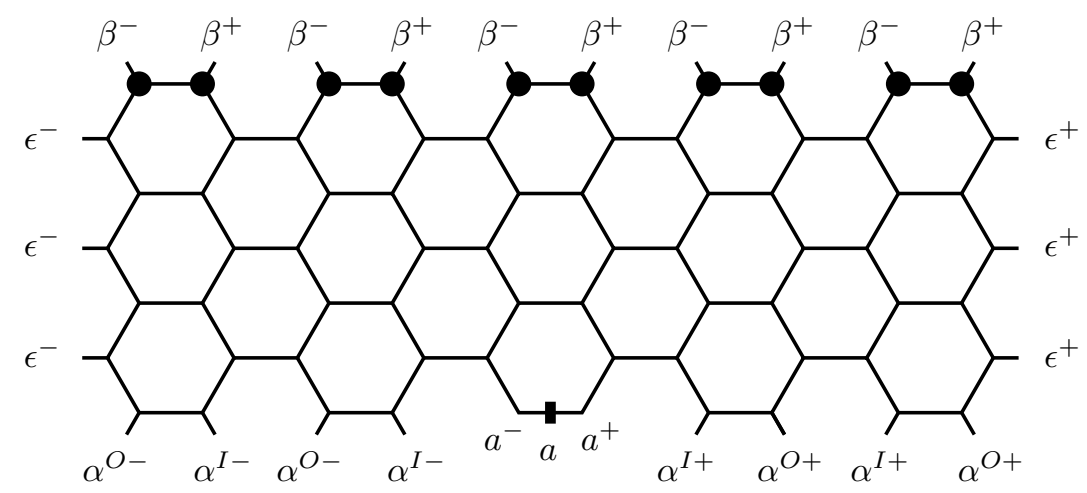

Fig. 3: The domain $D_{T, L}$ of height $T=7$ and width $2 L+1=9$, with the weighted vertices on the $\beta$ boundary indicated. The external mid-edges attached to $a^{-}$and $a^{+}$are present in the domain but will not play a part in the identity, and are thus not illustrated.

It is clear that if $y=1$ or if $v$ is not a weighted vertex, then the RHS of (4) disappears and thus (4) reduces to something very similar to $(3)$ - the differences being that here $a$ is not an external mid-edge, and $V^{\prime}\left(D_{T, L}\right)$ does not quite include all vertices in the domain. The factors $\mathrm{e}^{-\mathrm{i} \sigma(-\pi / 6)}$ and $\mathrm{e}^{-\mathrm{i} \sigma(\pi / 6)}$ are the contributions of the modified winding angles of walks to $\beta^{+}$and $\beta^{-}$mid-edges respectively.

\subsection{The domain identity}

In Duminil-Copin and Smirnov (2012), the authors use Lemma 2 to prove that the growth constant of selfavoiding walks (the dilute regime) is $x_{c}^{-1}=\sqrt{2+\sqrt{2}}$. They do so by considering a special trapezoidal domain, and using the local identity $(3)$ to derive a domain identity satisfied by generating functions of SAWs which end on different sides of the domain. In Beaton et al. (2012), that identity is generalised to one which relates generating functions of the $O(n)$ loop model and takes into account the surface fugacity $y$.

Here, we construct a similar identity to the one used in Beaton et al.(2012). We take $\sigma$ and $x_{\mathrm{c}}$ to be the values given in (2).

Define

$$
\begin{array}{ll}
A_{T, L}^{O}(x, y)=\sum_{\gamma: a \rightarrow \alpha^{O+} \cup \alpha^{O-}} x^{|\gamma|} y^{c(\gamma)} & A_{T, L}^{I}(x, y)=\sum_{\gamma: a \rightarrow \alpha^{I+} \bigcup \alpha^{I-}} x^{|\gamma|} y^{c(\gamma)} \\
E_{T, L}(x, y)=\sum_{\gamma: a \rightarrow \epsilon^{+} \bigcup \epsilon^{-}} x^{|\gamma|} y^{c(\gamma)} & B_{T, L}(x, y)=\sum_{\gamma: a \rightarrow \beta^{+} \bigcup \beta^{-}} x^{|\gamma|} y^{c(\gamma)},
\end{array}
$$

where each sum runs over SAWs which start at $a$ and end in the indicated set of external mid-edges of $D_{T, L}$. Also, define

$$
P_{T, L}(x, y)=\sum_{\rho \ni a} x^{|\rho|} y^{c(\rho)}
$$


which sums over all undirected (non-empty) self-avoiding polygons in $D_{T, L}$ which contain $a$. That is, $\rho$ is a simple closed loop on the edges of $D_{T, L}$ which passes through $a,|\rho|$ is the number of edges (or, equivalently, vertices) occupied by $\rho$ and $c(\rho)$ is the number of boundary vertices occupied by $\rho$.

Proposition 4 Let $T+L \equiv 1(\bmod 2)$. Then the generating functions $A_{T, L}^{O}, A_{T, L}^{I}, E_{T, L}, B_{T, L}$ and $P_{T, L}$, evaluated at $x=x_{\mathrm{c}}$, satisfy the identity

$$
c_{A}^{O} A_{T, L}^{O}\left(x_{\mathrm{c}}, y\right)+c_{A}^{I} A_{T, L}^{I}\left(x_{\mathrm{c}}, y\right)+c_{E} E_{T, L}\left(x_{\mathrm{c}}, y\right)+c_{P} P_{T, L}\left(x_{\mathrm{c}}, y\right)+c_{B}(y) B_{T, L}\left(x_{\mathrm{c}}, y\right)=c_{G},
$$

where

$$
\begin{aligned}
c_{A}^{O} & :=2 \cos \left(\frac{5 \pi}{16}\right)=\sqrt{2-\sqrt{2-\sqrt{2}}}, \quad c_{A}^{I}:=2 \cos \left(\frac{7 \pi}{16}\right)=\sqrt{2-\sqrt{2+\sqrt{2}}} \\
c_{E} & :=2 \cos \left(\frac{3 \pi}{16}\right)=\sqrt{2+\sqrt{2-\sqrt{2}}}, \\
c_{P} & :=\frac{4}{x_{\mathrm{c}}} \cos \left(\frac{7 \pi}{16}\right)=2 \sqrt{4+2 \sqrt{2}-\sqrt{2(10+7 \sqrt{2})}}, \\
c_{G} & :=4 x_{\mathrm{c}} \cos \left(\frac{\pi}{16}\right)=\sqrt{2(4-2 \sqrt{2}+\sqrt{2(2-\sqrt{2})})}, \text { and } \\
c_{B}(y) & :=2 \cos \left(\frac{\pi}{16}\right)-\frac{2\left(1-x_{\mathrm{c}} y-x_{\mathrm{c}}^{2} y^{2}\right) \cos \left(\frac{15 \pi}{16}\right)+2 x_{\mathrm{c}}^{2} y^{2} \cos \left(\frac{5 \pi}{16}\right)}{x_{\mathrm{c}} y\left(1+x_{\mathrm{c}} y\right)} \\
& =\frac{c_{B}}{x_{\mathrm{c}} y\left(1+x_{\mathrm{c}} y\right)}-\frac{x_{\mathrm{c}} y c_{A}^{O}}{1+x_{\mathrm{c}} y}, \quad \text { and } c_{B}:=c_{B}(1)=2 \cos \left(\frac{\pi}{16}\right)=\sqrt{2+\sqrt{2+\sqrt{2}}} .
\end{aligned}
$$

The proof follows by computing the sum

$$
S=\sum_{\substack{v \in V^{\prime}\left(D_{T, L}\right) \\ p, q, r \sim v}}(p-v) F^{*}(p)+(q-v) F^{*}(q)+(r-v) F^{*}(r),
$$

where $p, q, r$ are the three mid-edges adjacent to vertex $v$, in two ways. One one hand, the contribution to $S$ of any "internal" mid-edge (i.e. any mid-edge adjacent to two vertices in $V^{\prime}\left(D_{T, L}\right)$ ) will be 0 , and thus we only need to consider the contributions of external mid-edges. On the other hand, 4 guarantees that the contribution of any unweighted vertex is 0 , and so we can compute $S$ by calculating the contributions of the vertices on the $\beta$ boundary. We require $T+L \equiv 1(\bmod 2)$ so that we can pair up vertices on the $\beta$ boundary.

\section{The critical surface fugacity}

In this extended abstract we omit most of the technical results which enable us to adapt known results for the hypercubic lattice (see Hammersley et al. (1982) and van Rensburg et al. (2006)) to the honeycomb lattice, and instead only present the main result that we need. In a strip of height $T$, we set the mid-edge $a$ 


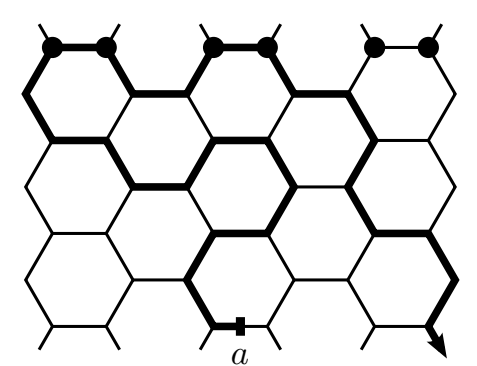

(a)

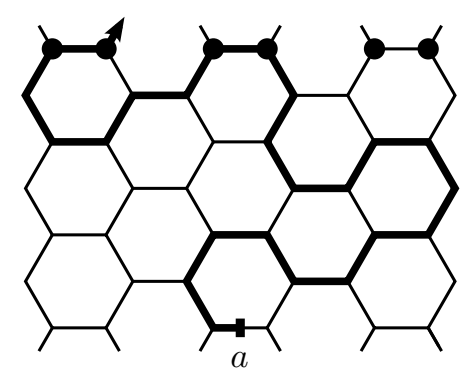

(b)

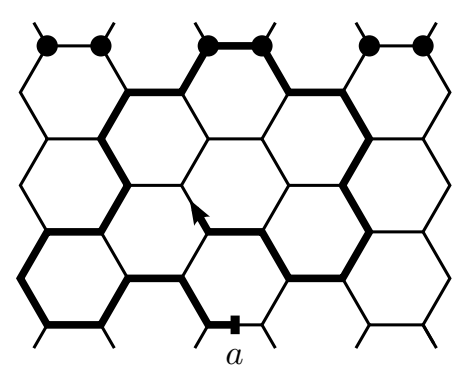

(c)

Fig. 4: Sections of the strip of height $T$, with (a) an arch, (b) a bridge and (c) a general walk. The dark circles indicate the weighted vertices on the top of the strip.

to be a horizontal mid-edge on the bottom of the strip (similar to its placement in the finite domain $D_{T, L}$ ). We then define the following three types of SAWs (see Figure 4): bridges, which start at $a$ and end at the top of the strip; arches, which start at $a$ and end on the bottom of the strip; and general walks, which start at $a$ and may end anywhere in the strip. We then define the generating function

$$
B_{T}(x, y)=\sum_{n, m \geq 0} b_{T, n}(m) x^{n} y^{m}
$$

where $B_{T, n}(m)$ is the number of length $n$ bridges in the strip of width $T$ which contain $m$ vertices at the top of the strip. We likewise define $A_{T}(x, y)$ and $C_{T}(x, y)$ for arches and general walks respectively.

The following proposition will allow us to relate the generating functions we considered in the previous section with the critical surface fugacity $y_{\mathrm{c}}$. Recall from Section 1 the definition of $\mu(y)$.

Proposition 5 For $y>0$, the generating functions $A_{T}(x, y), B_{T}(x, y)$ and $C_{T}(x, y)$ all have the same radius of convergence, $\rho_{T}(y)$. The sequence $\rho_{T}(y)$ decreases to $\rho(y):=\mu(y)^{-1}$ as $T \rightarrow \infty$. In particular, $\rho_{T}(y)$ decreases to $\rho:=\mu^{-1}$ for $y \leq y_{\mathrm{c}}$.

There exists a unique $y_{T}>0$ such that $\rho_{T}\left(y_{T}\right)=x_{\mathrm{c}}:=\mu^{-1}$. The series (in $\left.y\right) A_{T}\left(x_{\mathrm{c}}, y\right), B_{T}\left(x_{\mathrm{c}}, y\right)$ and $C_{T}\left(x_{\mathrm{c}}, y\right)$ have radius of convergence $y_{T}$, and $y_{T}$ decreases to the critical fugacity $y_{\mathrm{c}}$ as $T \rightarrow \infty$.

We now return to the identity (5) relating the generating functions in the domain $D_{T, L}$. Note that $c_{B}(y)$ is a continuous and monotone decreasing function of $y$ for $y>0$, and that $c_{B}\left(y^{\dagger}\right)=0$ where

$$
y^{\dagger}=\sqrt{\frac{2+\sqrt{2}}{1+\sqrt{2}-\sqrt{2+\sqrt{2}}}} .
$$

For $0<y<y^{\dagger}$, every term in 5 is non-negative. Observe that $A_{T, L}^{O}, A_{T, L}^{I}, B_{T, L}$ and $P_{T, L}$ are increasing with $L$. (As $L$ increases these generating functions just count more and more objects.) We then see that for those values of $L$ satisfing $T+L \equiv 1(\bmod 2), E_{T, L}$ must decrease as $L$ increases. It is thus valid to take the limit $L \rightarrow \infty$ of [5] over the values of $L$ with $T+L \equiv 1(\bmod 2)$. But now $A_{T, L}^{O}, A_{T, L}^{I}$, $B_{T, L}$ and $P_{T, L}$ actually increase with $L$ regardless of whether $T+L \equiv 1(\bmod 2)$ or not, and so they have the same limits as $L \rightarrow \infty$ over any subsequence of $L$ values. Hence, we can in fact take the limit 
$L \rightarrow \infty$ of (5) over all values of $L$. If we define

$$
A_{T}^{O}\left(x_{\mathrm{c}}, y\right):=\lim _{L \rightarrow \infty} A_{T, L}^{O}\left(x_{\mathrm{c}}, y\right),
$$

and similar limits for the other generating functions (we also have $\lim _{L \rightarrow \infty} B_{T, L}(x, y)=B_{T}(x, y)$ as defined earlier), then we obtain

$$
c_{A}^{O} A_{T}^{O}\left(x_{\mathrm{c}}, y\right)+c_{A}^{I} A_{T}^{I}\left(x_{\mathrm{c}}, y\right)+c_{E} E_{T}\left(x_{\mathrm{c}}, y\right)+c_{P} P_{T}\left(x_{\mathrm{c}}, y\right)+c_{B}(y) B_{T}\left(x_{\mathrm{c}}, y\right)=c_{G} .
$$

In this rest of this section, we will prove the following:

Proposition 6 If it can be shown that

$$
B\left(x_{\mathrm{c}}, 1\right):=\lim _{T \rightarrow \infty} B_{T}\left(x_{\mathrm{c}}, 1\right)=0
$$

then $y_{\mathrm{c}}=y^{\dagger}$.

The proof that $B\left(x_{\mathrm{c}}, 1\right)=0$ is quite involved and will thus be omitted from this extended abstract; see the appendix of Beaton(2012).

We begin by establishing a lower bound on $y_{\mathrm{c}}$ with a straightforward corollary to Proposition 5 .

Corollary 7 The critical surface fugacity $y_{\mathrm{c}}$ satisfies

$$
y_{\mathrm{c}} \geq y^{\dagger} .
$$

Proof: For $y<y^{\dagger}$ the identity (7) establishes the finiteness of $B_{T}\left(x_{\mathrm{c}}, y\right)$, and thus we see $y_{T} \geq y^{\dagger}$. By Proposition 5 it then follows that $y_{\mathrm{c}} \geq y^{\dagger}$.

We now show that one of the generating functions in (7) has disappeared in the limit $L \rightarrow \infty$.

Corollary 8 For $0 \leq y<y^{\dagger}$,

$$
E_{T}\left(x_{\mathrm{c}}, y\right):=\lim _{L \rightarrow \infty} E_{T, L}\left(x_{\mathrm{c}}, y\right)=0
$$

and hence

$$
c_{A}^{O} A_{T}^{O}\left(x_{\mathrm{c}}, y\right)+c_{A}^{I} A_{T}^{I}\left(x_{\mathrm{c}}, y\right)+c_{P} P_{T}\left(x_{\mathrm{c}}, y\right)+c_{B}(y) B_{T}\left(x_{\mathrm{c}}, y\right)=c_{G} .
$$

Proof: By Proposition 5, $y_{T}$ is the radius of convergence of $C_{T}\left(x_{\mathrm{c}}, y\right)$. Since $y_{T} \geq y_{\mathrm{c}} \geq y^{\dagger}$, it follows that $C_{T}\left(x_{\mathrm{c}}, y\right)$ is convergent for $0 \leq y<y^{\dagger}$. Now

$$
\sum_{L} E_{T, L}\left(x_{\mathrm{c}}, y\right) \leq C_{T}\left(x_{\mathrm{c}}, y\right)<\infty,
$$

as each walk counted by $E_{T, L}$, for every value of $L$, will also be counted by $C_{T}$. The corollary follows immediately.

We note here that $A_{T}^{O}\left(x_{\mathrm{c}}, y\right) \leq C_{T}\left(x_{\mathrm{c}}, y\right)$ (since any walk counted by $A_{T}^{O}$ is also counted by $C_{T}$ ), and likewise for $A_{T}^{O}$ and $P_{T}$. Hence all the generating functions featured in (8) have radius of convergence at least $y_{T}$. 


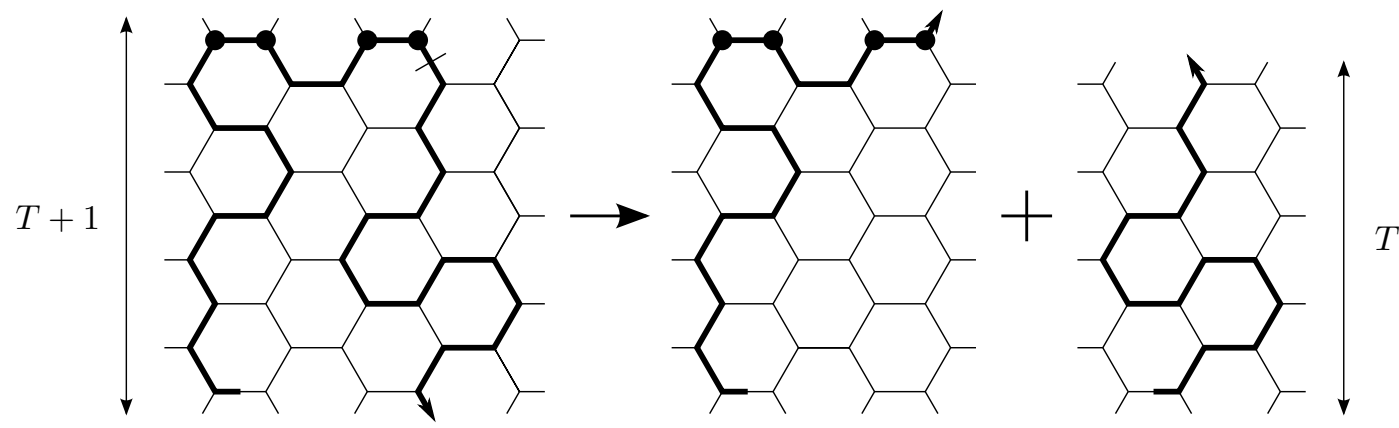

Fig. 5: Factorisation of a walk counted by $A_{T+1}^{O}$ into two bridges.

Now consider the $y=1$ case of 8 :

$$
c_{A}^{O} A_{T}^{O}\left(x_{\mathrm{c}}, 1\right)+c_{A}^{I} A_{T}^{I}\left(x_{\mathrm{c}}, 1\right)+c_{P} P_{T}\left(x_{\mathrm{c}}, 1\right)+c_{B} B_{T}\left(x_{\mathrm{c}}, 1\right)=c_{G} .
$$

Since $A_{T}^{O}\left(x_{\mathrm{c}}, 1\right), A_{T}^{I}\left(x_{\mathrm{c}}, 1\right)$ and $P_{T}\left(x_{\mathrm{c}}, 1\right)$ all increase with $T$ (as $T$ increases these generating functions count more and more objects), and since they are all bounded by this identity, it follows that they all have limits as $T \rightarrow \infty$. Then $B_{T}\left(x_{\mathrm{c}}, 1\right)$ must decrease as $T$ increases, and it too has a limit as $T \rightarrow \infty$. As indicated in Proposition 6, we denote this limit

$$
B\left(x_{\mathrm{c}}, 1\right):=\lim _{T \rightarrow \infty} B_{T}\left(x_{\mathrm{c}}, 1\right) .
$$

Proof of Proposition 6: Assume now that $B\left(x_{\mathrm{c}}, 1\right)=0$. Any walk counted by $A_{T+1}^{O}\left(x_{\mathrm{c}}, y\right)$ which has contacts with the top boundary can be factored into two pieces by cutting it at the mid-edge immediately following its last surface contact. (See Figure 5.) The first piece, after reflecting the last step, is an object counted by $B_{T+1}\left(x_{\mathrm{c}}, y\right)$, while the second piece (with its direction reversed) will be counted by $\left(1+x_{\mathrm{c}}\right) B_{T}\left(x_{\mathrm{c}}, 1\right) / 2$. Thus we obtain

$$
\begin{aligned}
A_{T+1}^{O}\left(x_{\mathrm{c}}, y\right)-A_{T}^{O}\left(x_{\mathrm{c}}, 1\right) & \leq \frac{1+x_{\mathrm{c}}}{2} \cdot B_{T+1}\left(x_{\mathrm{c}}, y\right) B_{T}\left(x_{\mathrm{c}}, 1\right) \\
& \leq B_{T+1}\left(x_{\mathrm{c}}, y\right) B_{T}\left(x_{\mathrm{c}}, 1\right) .
\end{aligned}
$$

This inequality is valid in the domain of convergence of the series it involves, that is, for $y<y_{T+1}$. Using similar arguments we can obtain the equivalent inequality for $A_{T+1}^{I}\left(x_{\mathrm{c}}, y\right)$ and $P_{T+1}\left(x_{\mathrm{c}}, y\right)$.

Combining this decomposition for $A_{T+1}^{O}, A_{T+1}^{I}$ and $P_{T+1}$, we find for $0 \leq y<y_{T+1}$,

$$
\begin{array}{r}
c_{A}^{O}\left[A_{T+1}^{O}\left(x_{\mathrm{c}}, y\right)-A_{T}^{O}\left(x_{\mathrm{c}}, 1\right)\right]+c_{A}^{I}\left[A_{T+1}^{I}\left(x_{\mathrm{c}}, y\right)-A_{T}^{I}\left(x_{\mathrm{c}}, 1\right)\right]+c_{P}\left[P_{T+1}\left(x_{\mathrm{c}}, y\right)-P_{T}\left(x_{\mathrm{c}}, 1\right)\right] \\
\leq\left(c_{A}^{O}+c_{A}^{I}+c_{P}\right) B_{T+1}\left(x_{\mathrm{c}}, y\right) B_{T}\left(x_{\mathrm{c}}, 1\right) .
\end{array}
$$

Using (8) to eliminate the $A^{O}, A^{I}$ and $P$ terms, we obtain

$$
c_{B} B_{T}\left(x_{\mathrm{c}}, 1\right)-c_{B}(y) B_{T+1}\left(x_{\mathrm{c}}, y\right) \leq\left(c_{A}^{O}+c_{A}^{I}+c_{P}\right) B_{T+1}\left(x_{\mathrm{c}}, y\right) B_{T}\left(x_{\mathrm{c}}, 1\right),
$$


and hence

$$
0 \leq \frac{1}{B_{T+1}\left(x_{\mathrm{c}}, y\right)} \leq \frac{\left(c_{A}^{O}+c_{A}^{I}+c_{P}\right)}{c_{B}}+\frac{c_{B}(y)}{c_{B} B_{T}\left(x_{\mathrm{c}}, 1\right)}
$$

In particular, for $0 \leq y<y_{\mathrm{c}}=\lim _{T \rightarrow \infty} y_{T}$ and for any $T$,

$$
0 \leq \frac{x_{\mathrm{c}}\left(c_{A}^{O}+c_{A}^{I}+c_{P}\right)}{c_{B}}+\frac{c_{B}(y)}{c_{B} B_{T}\left(x_{\mathrm{c}}, 1\right)} .
$$

Now consider what happens as $T \rightarrow \infty$. By assumption, $\lim _{T \rightarrow \infty} B_{T}\left(x_{\mathrm{c}}, 1\right)=0$. Suppose (for a contradiction) that $y_{\mathrm{c}}>y^{\dagger}$. Then for any $y^{\dagger}<y<y_{\mathrm{c}}$ and sufficiently large $T$, the RHS of (11) will be negative, because $c_{B}(y)<0$ for $y>y^{\dagger}$ and $B_{T}\left(x_{\mathrm{c}}, 1\right)^{-1}$ will become arbitrarily large. This contradicts the inequality, and we are forced to conclude $y_{\mathrm{c}} \leq y^{\dagger}$, and hence $y_{\mathrm{c}}=y^{\dagger}$.

\section{Acknowledgements}

I thank Murray Batchelor for suggesting this problem, and Tony Guttmann for helpful conversations. I received support from the ARC Centre of Excellence for Mathematics and Statistics of Complex Systems (MASCOS), as well as the Australian Mathematical Society (AustMS) in the form of a Lift-Off Fellowship. Part of this work was carried out while I was a guest of the Mathematical Sciences Research Institute (MSRI) in Berkeley, CA, during the Spring 2012 Random Spatial Processes Program, and I thank the Institute for its hospitality and the NSF (grant DMS-0932078) for its financial support.

\section{References}

M. T. Batchelor and C. M. Yung. Exact results for the adsorption of a flexible self-avoiding polymer chain in two dimensions. Phys. Rev. Lett., 74(11):2026-2029, 1995.

M. T. Batchelor, D. Bennett-Wood, and A. L. Owczarek. Two-dimensional polymer networks at a mixed boundary: Surface and wedge exponents. Eur. Phys. J. B, 5(1):139-142, 1998.

N. R. Beaton. The critical surface fugacity for self-avoiding walks on a rotated honeycomb lattice. Preprint, arXiv:1210.0274, 2012.

N. R. Beaton, M. Bousquet-Mélou, J. de Gier, H. Duminil-Copin, and A. J. Guttmann. The critical fugacity for surface adsorption of self-avoiding walks on the honeycomb lattice is $1+\sqrt{2}$. Preprint, arXiv:1109.0358, 2012.

H. Duminil-Copin and A. Hammond. Self-avoiding walk is sub-ballistic. Preprint, arXiv:1205.0401, 2012.

H. Duminil-Copin and S. Smirnov. The connective constant of the honeycomb lattice equals $\sqrt{2+\sqrt{2}}$. Ann. of Math., 175(3):1653-1665, 2012.

P. J. Flory. The configuration of real polymer chains. J. Chem. Phys., 17(3):303-310, 1949.

J. M. Hammersley, G. M. Torrie, and S. G. Whittington. Self-avoiding walks interacting with a surface. J. Phys. A: Math. Gen., 15(2):539-571, 1982. 
N. Madras and G. Slade. The Self-Avoiding Walk. Probability and its Applications. Birkhäuser, Boston, MA, 1993.

B. Nienhuis. Exact critical point and critical exponents of $O(n)$ models in two dimensions. Phys. Rev. Lett., 49(15):1062-1065, 1982.

W. J. C. Orr. Statistical treatment of polymer solutions at infinite dilution. Trans. Faraday Soc., 43:12-27, 1947.

S. Smirnov. Discrete complex analysis and probability. In Proceedings of the International Congress of Mathematicians, vol. I, pages 595-621, Hyderabad, India, 2010. Hindustan Book Agency.

E. J. J. van Rensburg, E. Orlandini, and S. G. Whittington. Self-avoiding walks in a slab: rigorous results. J. Phys. A: Math. Gen., 39(45):13869-13902, 2006. 